بررسى تأثير زنجبيل بر ديسمنوره اوليه در دانشجويان دانشخاه علوم بزشكى قزوين

حميده پاكنيت'، عزتالسادات حاجى سيدجوادى '*، زهرا كلمحمدى'، مهسا اشرفى'

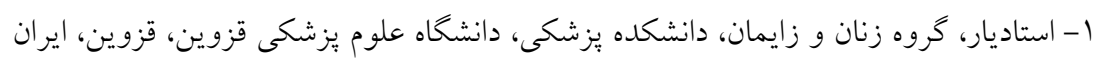

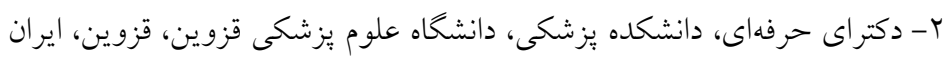

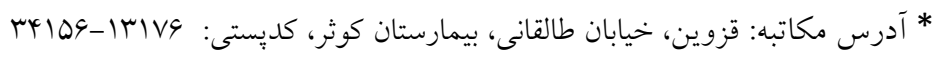

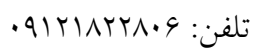

بـ

doi: $10.29252 / \mathrm{jmp} .4 .72 .98$

تاريخ تصويب: TV/O/IV

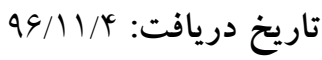

جكيده

مقدمه: ديسمنوره يكى مشكل اساسى در سلامت عمومى و فردى زنان مى باشد. با توجه به شيوع بالاى ديسمنوره اخيراً روشهاى



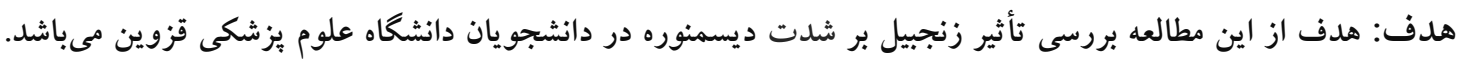

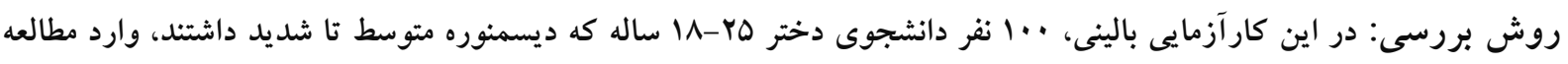

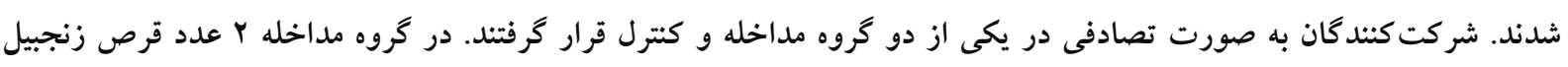

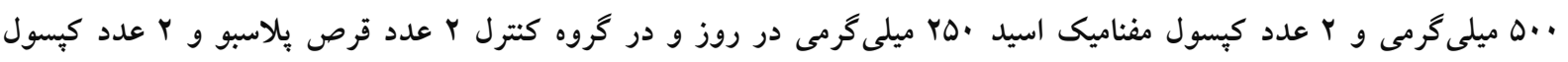

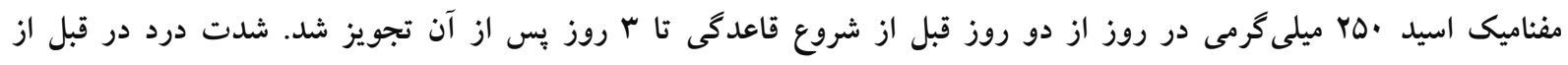

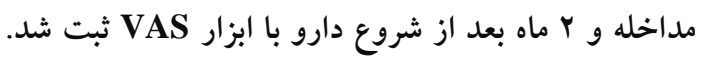

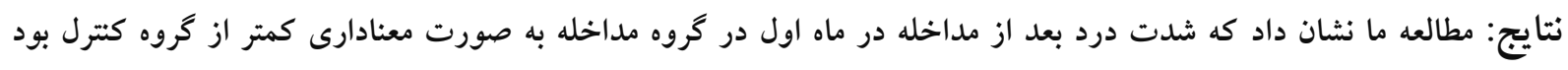
(Y)

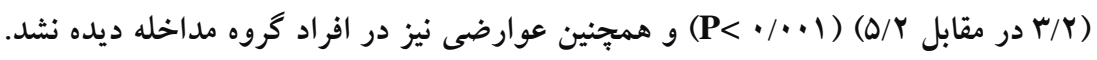

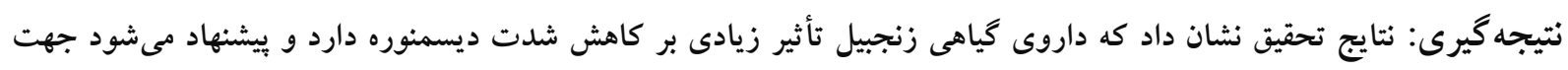

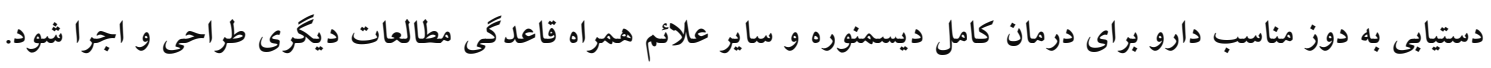

كل واز كان: زنجبيل، ديسمنوره، درد 
اهداف درمان، كاهش ميزان يروستاكلاندينها مىباشد [11)

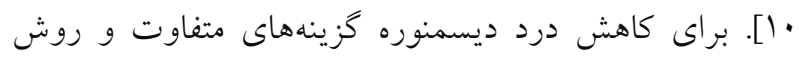

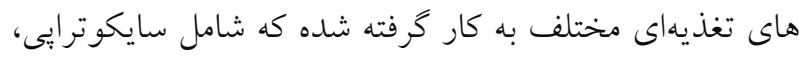

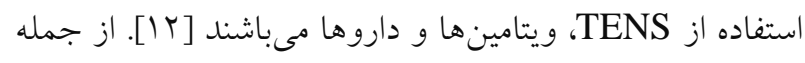

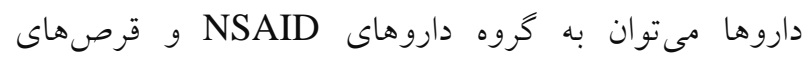

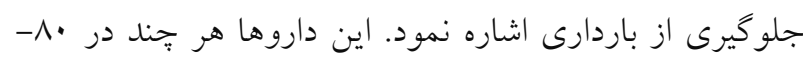

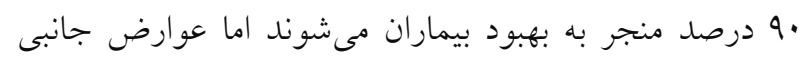

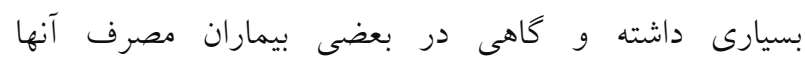

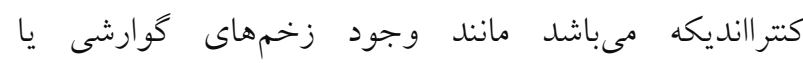

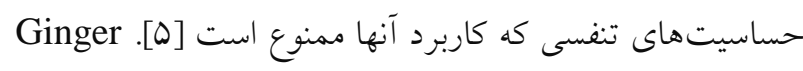
(زنجبيل) تاريخجه قدى قديمى در درمان بسيارى از بيمارىها

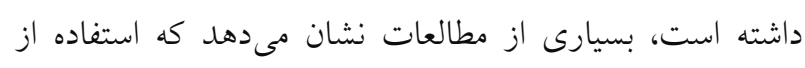

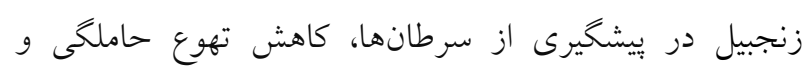

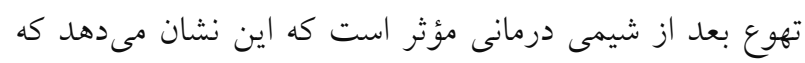

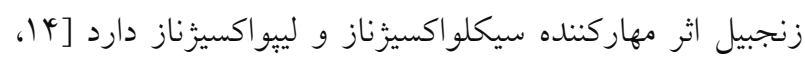

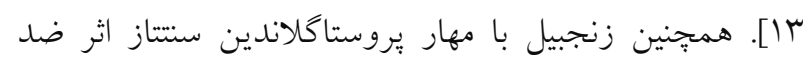

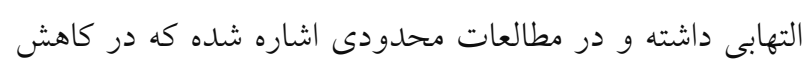
شدت ديسمنوره مؤثر است [10]

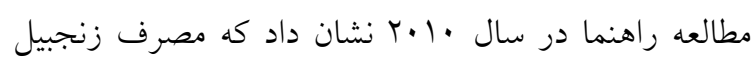

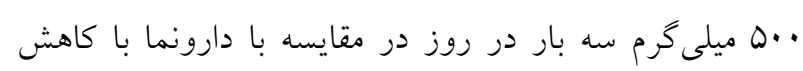

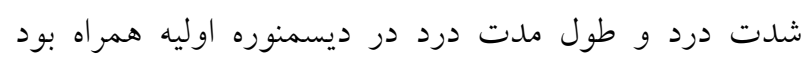

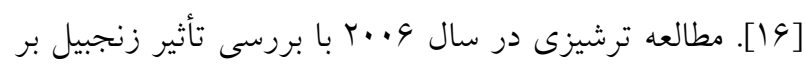

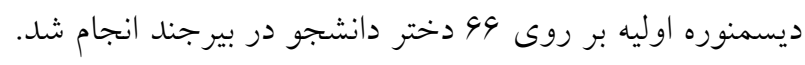

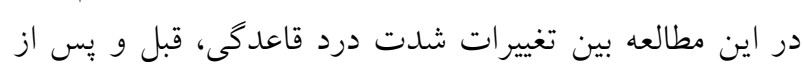

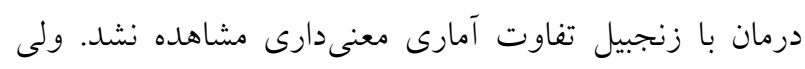

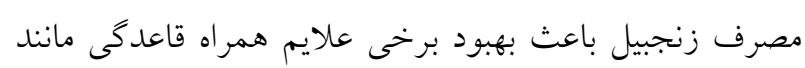

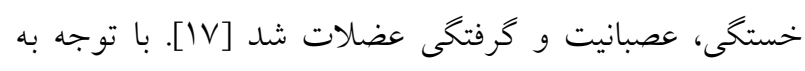

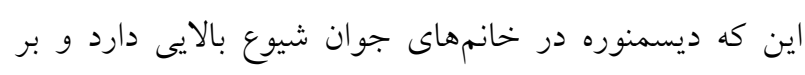

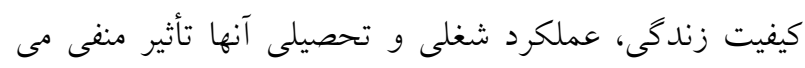

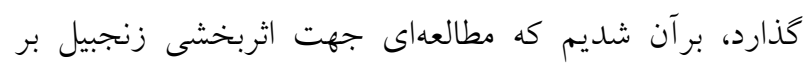
شدت ديسمنوره انجام دهيم.
ديسمنوره يك مشكل اساسى در سلامت عمومى و فردى

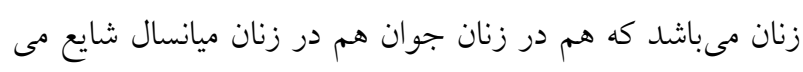

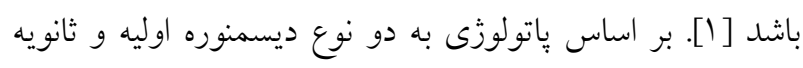

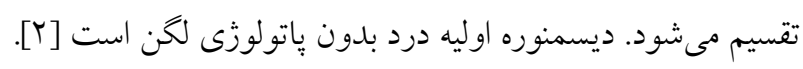

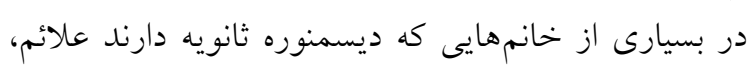

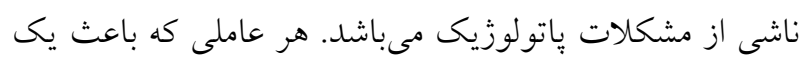

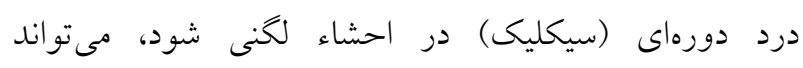

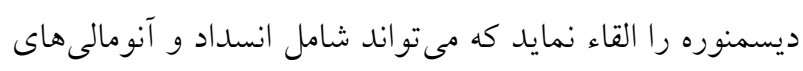

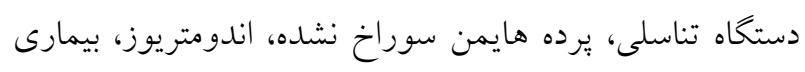

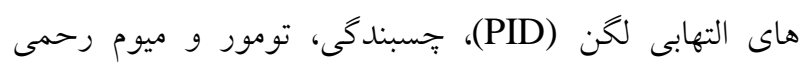

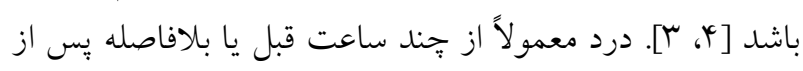

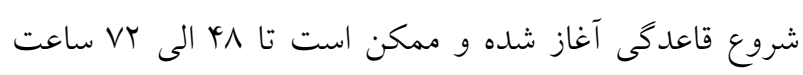

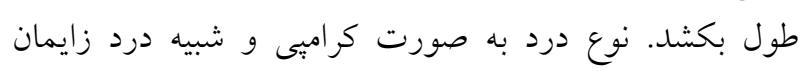
است و كاهى به قسمتهايى از قدام ران انتشار مىيابد [ب].

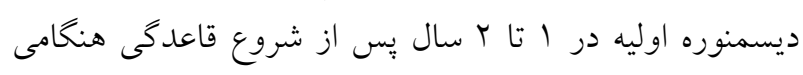

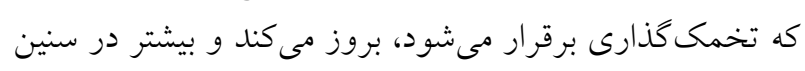

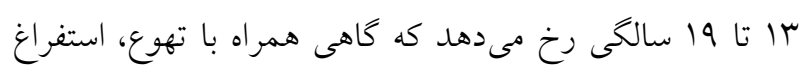

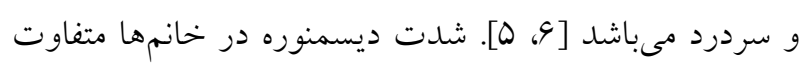

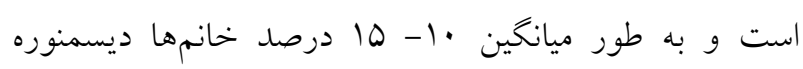

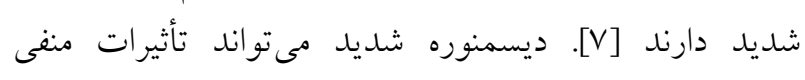

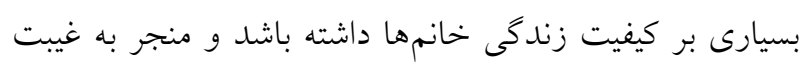

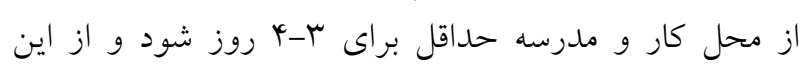

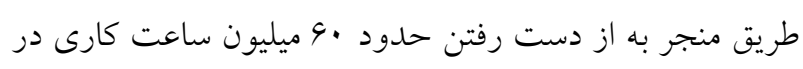

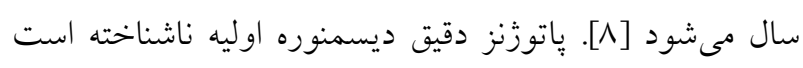

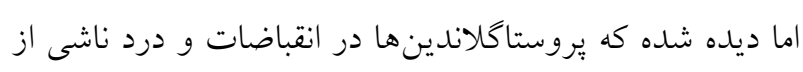

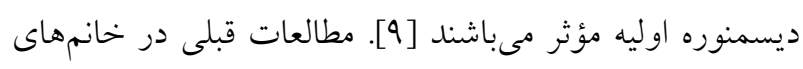

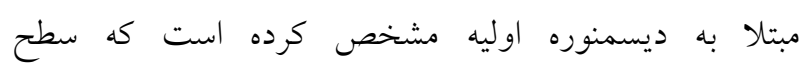

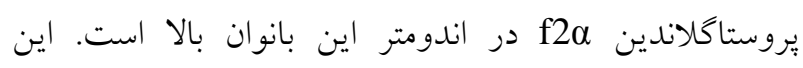

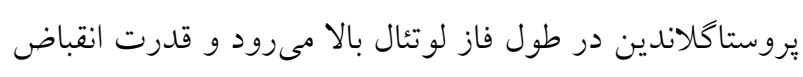

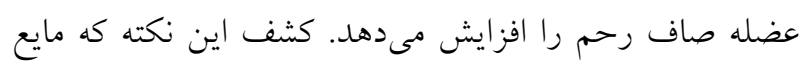

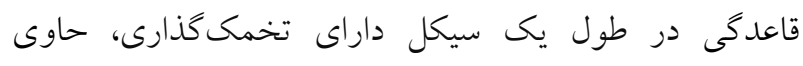

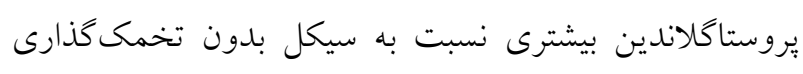

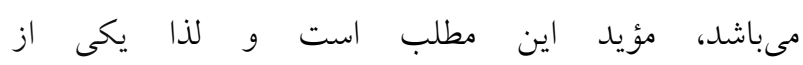

\section{4 شماره مسلسل هفتاد و دوم، هاييز يوها}


در پِاكتهاى يكسان و بسته شده تحويل شركت كنندگان داده

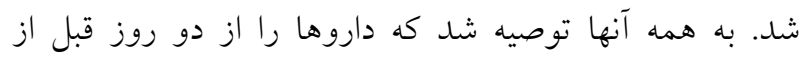

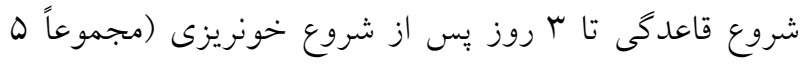

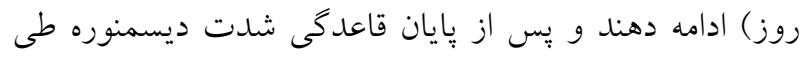

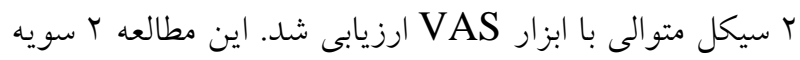
كور بود و مجرى و افرادى كه دارو دريافت كردند از محتويات داخل بِاكت ها اطلاعى نداشتند.

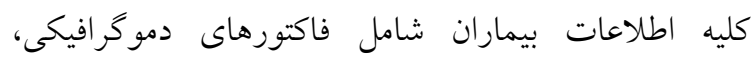
اطلاعات قاعدگى و شدت درد در خكى ليست ساخته شده توسط مجرى ثبت شد و وارد نرمافزار

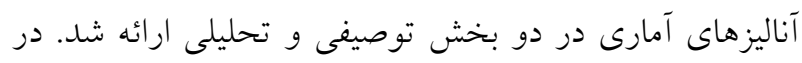

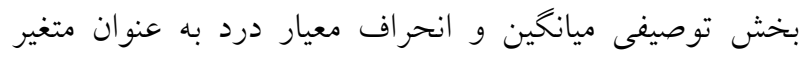

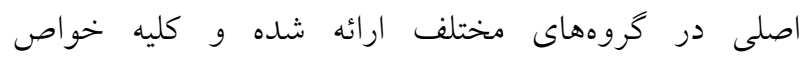

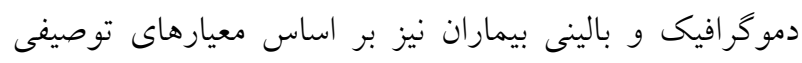

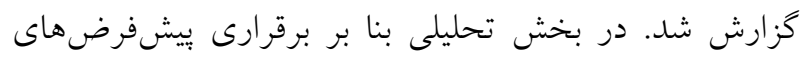

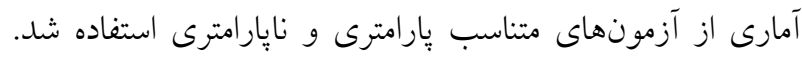

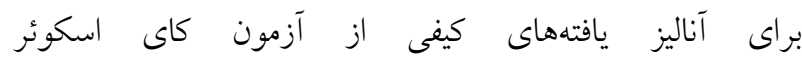
و براى مقايسه دادههاى كمى از آزمون (CHI- SQUARE) مستقل استفاده شد. در صورت برقرار نبودن مفروضات اوليه هماند نرمال بودن از آزمون نايارامترى من - ويتنى استفاده شدر.

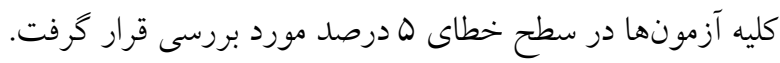

\section{نتايج}

از •النفرى كه وارد مطالعه شدند •النفر به علل مختلف



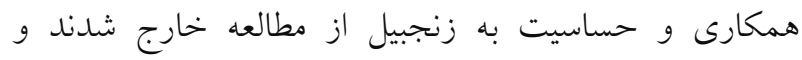

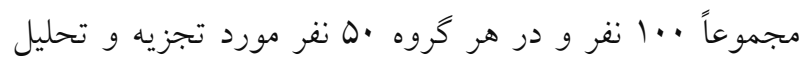

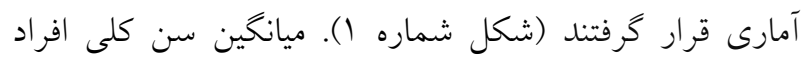

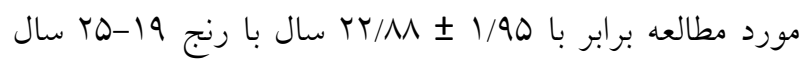

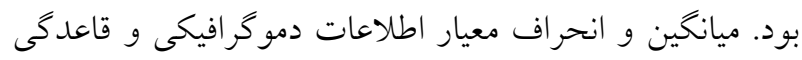

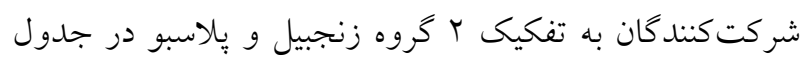

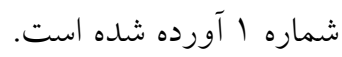

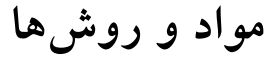

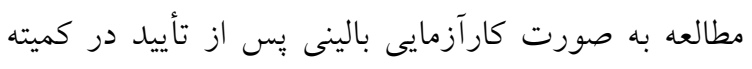

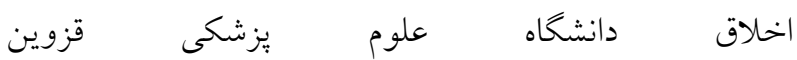
و ثبت در مركز كارآزمايى (IR.QUMS.REC.1395.205)

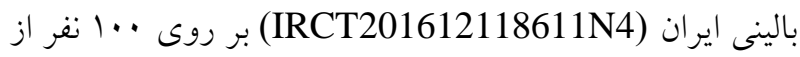

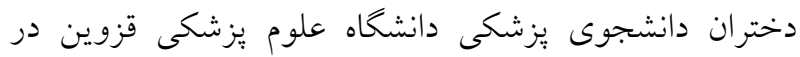

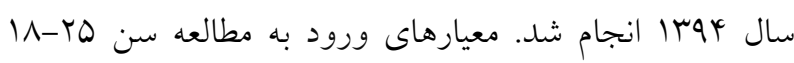

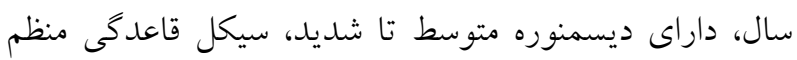

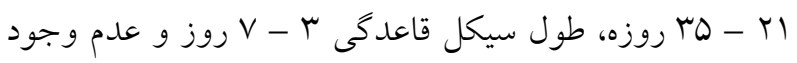

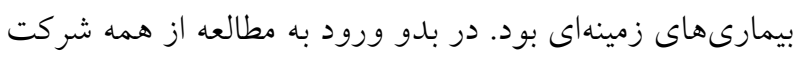

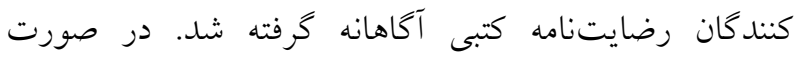

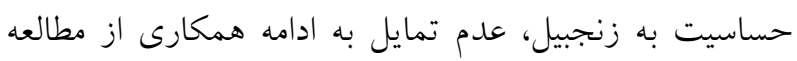
حذف شدنل. در زمان ورود به مطالعه يرسشنامهاى كه حاوى اطلاعات فردى شامل سن، وزن، قد، رشته تحصيلى، شغل و تحصيلات ونهات

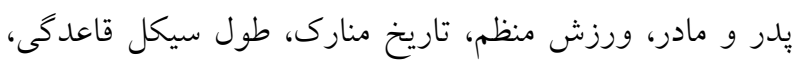

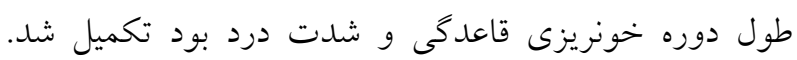
شدت ديسمنوره با مقياس آنالوگ بصرى ( pain visual (analougu scale

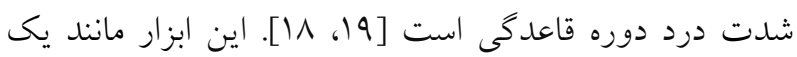

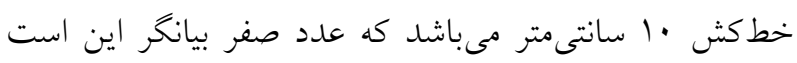

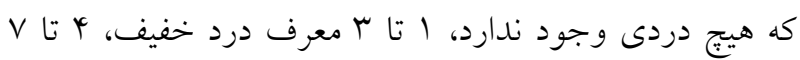
1. نشانكر درد متوسط و در صورت نشان دادن عدد 1 تا

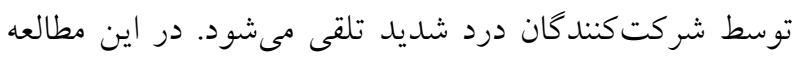

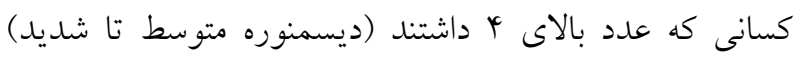

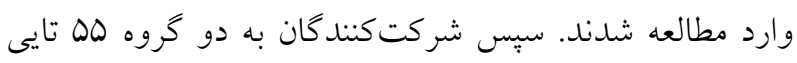

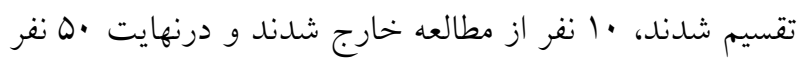

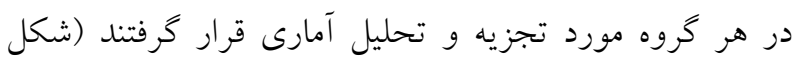

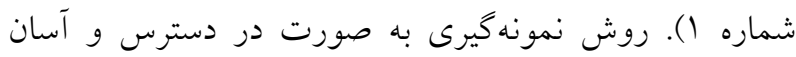

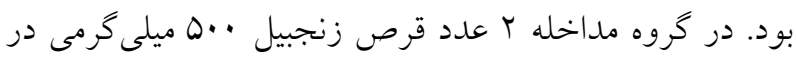
روز ساخت شركت دينه قزوين و r عدد كيسول مفناميك اسيد

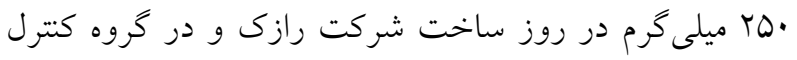

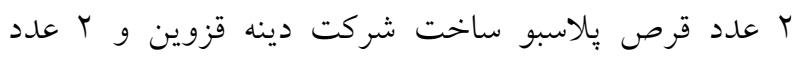

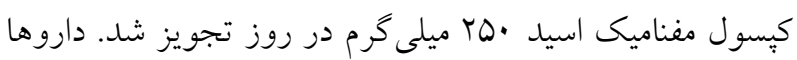


بررسى تأثير زنجبيل ...

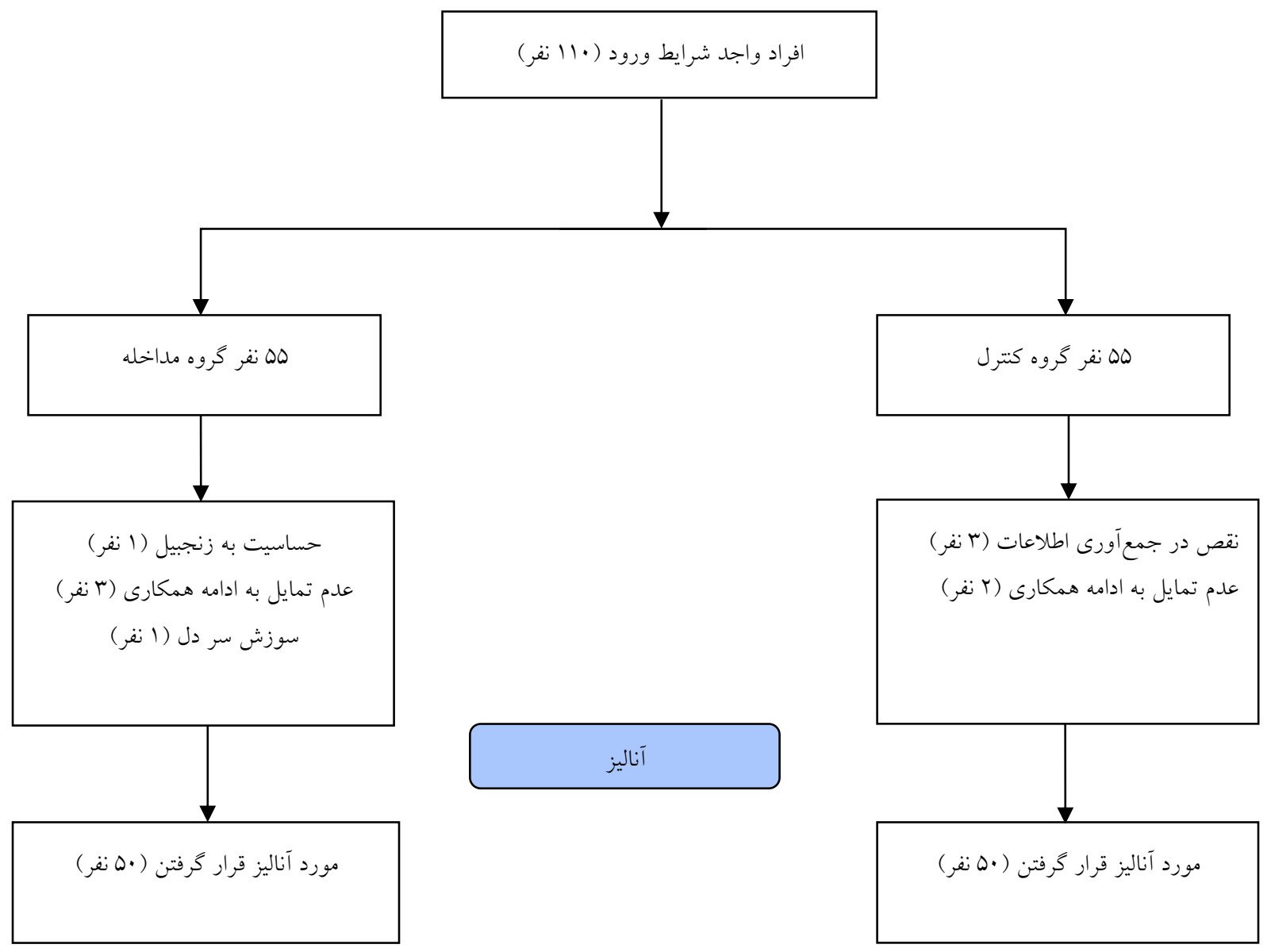

شكل شماره ا- فلوجارت مطالعه (CONSORT format)

\begin{tabular}{|c|c|c|c|}
\hline P-value & ميانگين土انحر اف معيار & ميانگين انحر اف معيار & متغير \\
\hline$\cdot / \pi t$ & $r r / I r \pm r / \cdot V$ & $Y Y / G Y \pm I / \Lambda$ & سن (سال) \\
\hline$\cdot / 19 \mathrm{~V}$ & $1 G \Gamma / \Gamma 9 \pm Y / T \Delta$ & $19 r / 0 r \pm r / \cdot 1$ & قد (سانتىمتر) \\
\hline$\cdot / 490$ & $\Delta V / T \pm \Delta / N{ }^{4}$ & $0 N / 99 \pm 9 / 49$ & 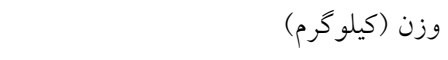 \\
\hline$\cdot / 9 \cdot 0$ & $r \mid / \Psi V \pm r / 41$ & $r I / V \Lambda \pm T / M r$ & $(\mathrm{~kg} / \mathrm{m} 2) \mathrm{BMI}$ \\
\hline 1 & $\mid r / \Delta T \pm \cdot / 90$ & $|r / \Delta T \pm| / \mid r$ & سن منارى (سال) \\
\hline$\cdot / 019$ & $T V / D G \pm I / T Y$ & $T V / Y Y \pm T / T D$ & مدت زمان سيكل قاعدكى (روز) \\
\hline$\cdot / \pi T V$ & $9 / \pi Y \pm 1 / \cdot V$ & $Q / 9 \wedge \pm 1 / \cdot 0$ & مدت زمان خونريزى (روز) \\
\hline.$/ 914$ & 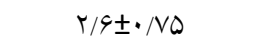 & $r / \Delta T \pm \cdot / \Lambda I$ & مدت زمان وجود علائم ديسمنوره (روز) \\
\hline
\end{tabular}


همجنين در طول زمان كاهش نمره درد در كروه مداخله

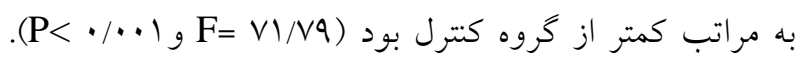

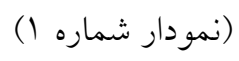

با توجه به دوز مصرفى زنجبيل عوارضى نظير سوزش سر الر

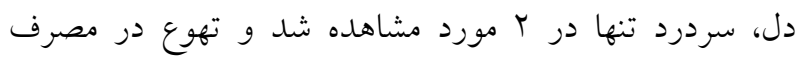

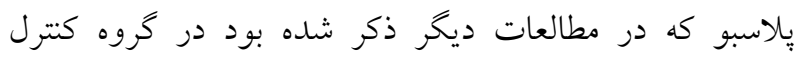

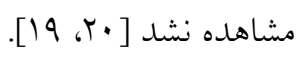

همانطور كه مشاهده مى شود ميانخين متغيرهاى

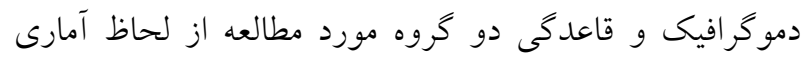

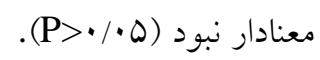

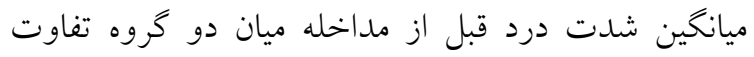

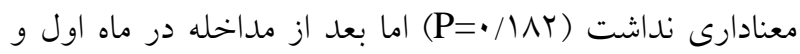
دوم در گروه مداخله به صورت معنادارى كمتر از خروه كنترل

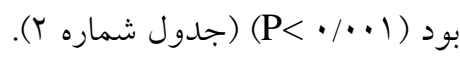

جدول شماره Y- ميانكين و انحراف معيار شدت درد در قبل و بعد از مداخله در دو كروه

\begin{tabular}{|c|c|c|c|c|c|}
\hline P-value 2 & $\mathbf{F}$ & P-value 1 & ميانگين土انحر اف معيار & 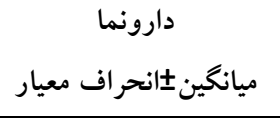 & زمان \\
\hline \multirow{3}{*}{$<\cdot / \cdot \cdot 1$} & & . /NAT & $9 N Y \pm \cdot / 99$ & $V / \cdot \pm 1 / l$. & قبل از مداخله \\
\hline & VI/Va & $<\cdot / \cdot \cdot 1$ & $\Gamma / N T \pm I / r \wedge$ & $\Delta / 4 \varphi \pm 1 / 4$. & ماه اول \\
\hline & & $<\cdot / \cdot \cdot 1$ & $r / T \pm 1 / r V$ & $\Delta / T \pm 1 / N Y$ & ماه دوم \\
\hline
\end{tabular}

P-value : Independent t test

P-value 2: ANOVA repeated measure

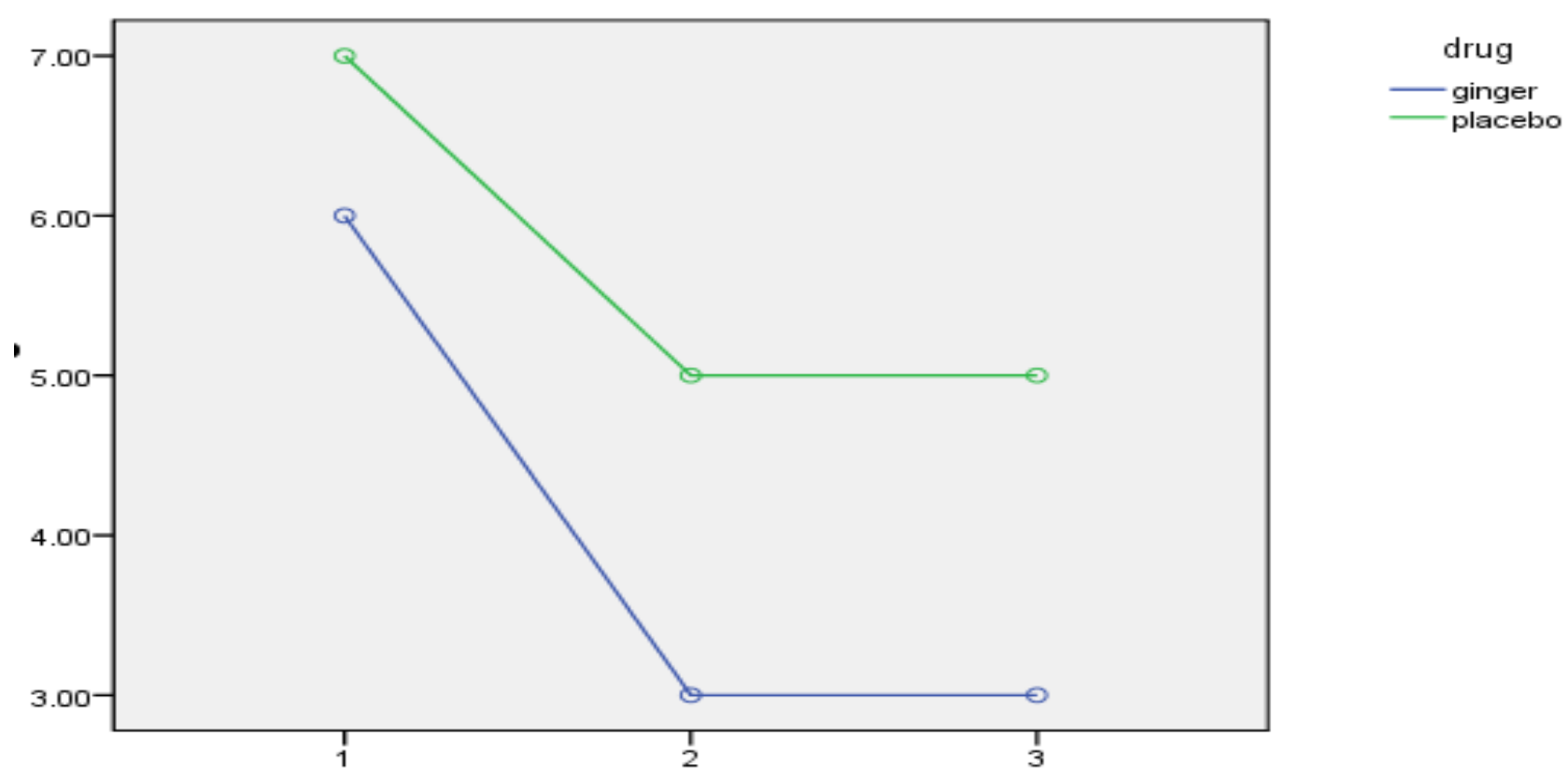

نمودار شماره ا-ميانگين درد در زمانهاى مختلف در دو گروه مداخله و كنترل 
ساكن خوابكاههاى علوم يزشكى اصفهان و شهيد بهشتى تهران

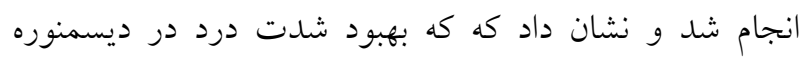

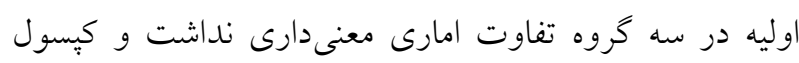
زنجبيل همانند دو داروى شيميايى مرسوم در درمان ديسمنوره

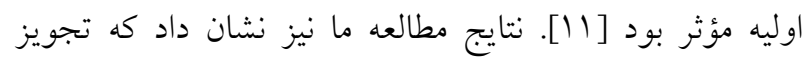

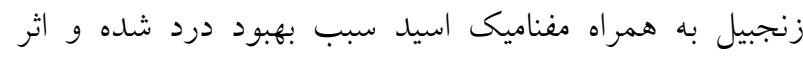

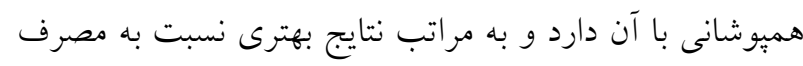

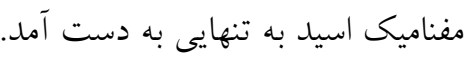

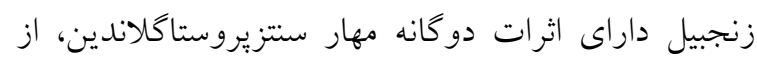

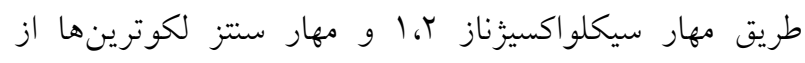

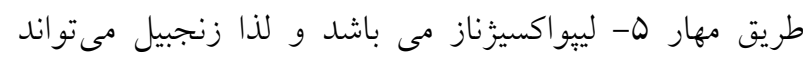

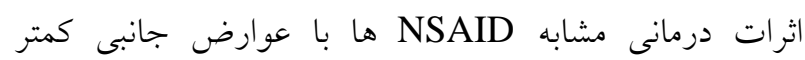

داشته باشد [ [T]].

در مطالعهاى كه توسط داوودآبادى و همكاران به منظور

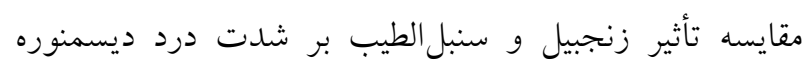

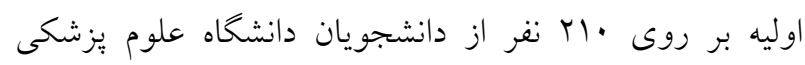

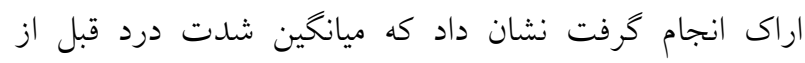
مصرف دارو در سه گروه زنجبيل و سنبل الطيب و كنترل،

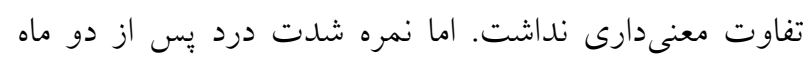

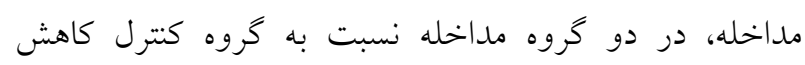

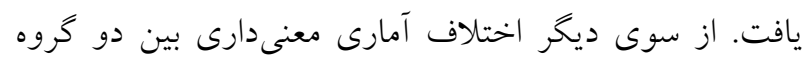

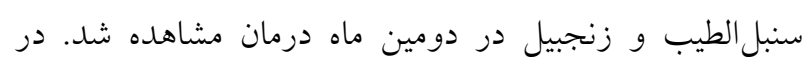

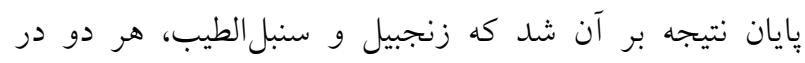

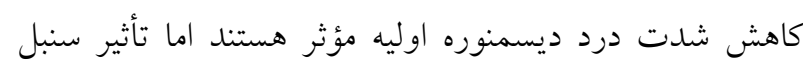

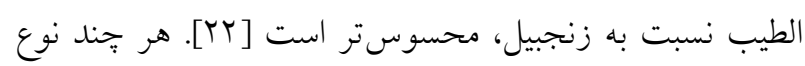

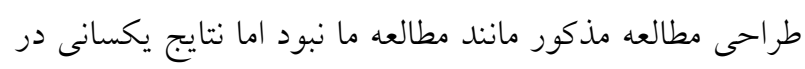

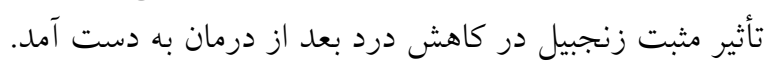

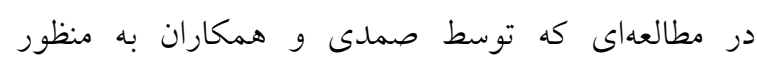

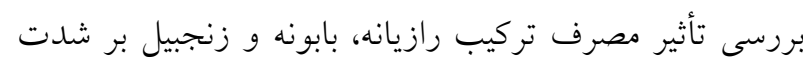

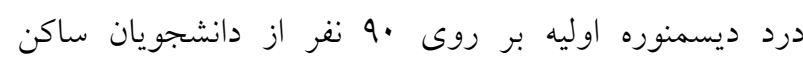

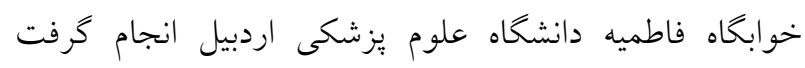

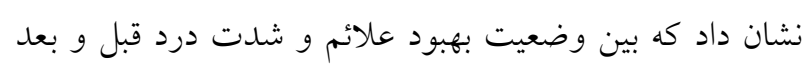

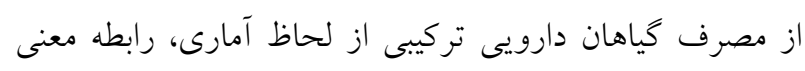

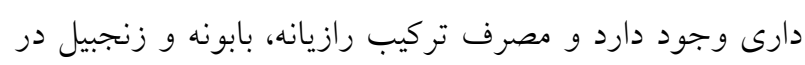

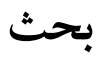

نتايج مطالعه ما نشان داد كه ميانخين درد قبل از مداخله

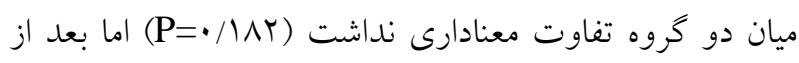

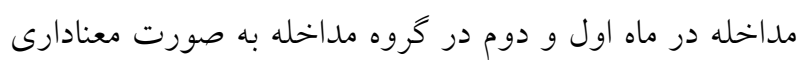

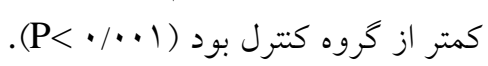

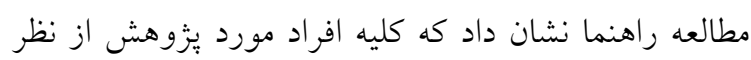

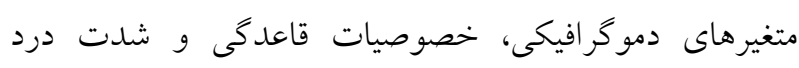

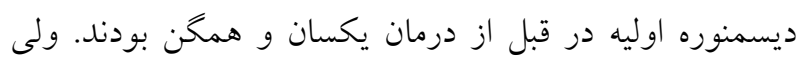
يس إز مداخله شدت درد در ديسمنوره اوليه در گروه زنجبيل

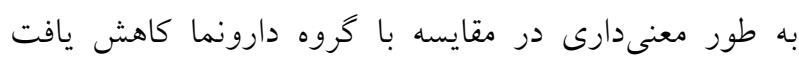
[19]. نتايج مطالعه مذكور همسو با يافتهاى مطاى مطالعه ما بود.

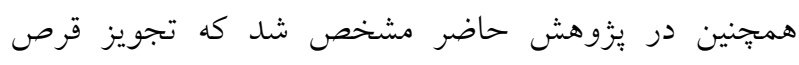

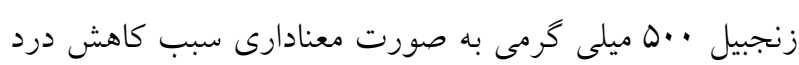

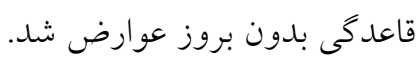
مطالعه ترشيزى به صورت كارآزمايى بالينى بر روى برو عو 99

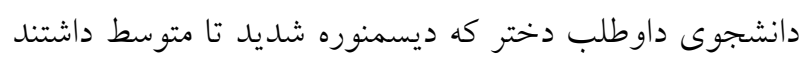

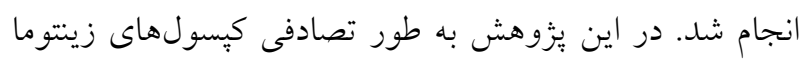

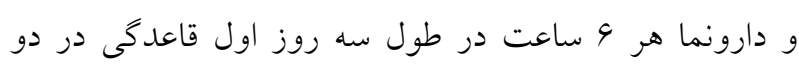

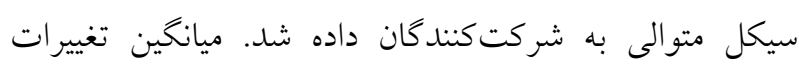

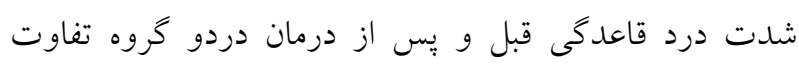

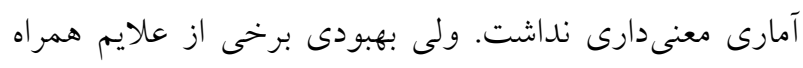

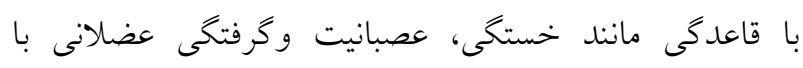

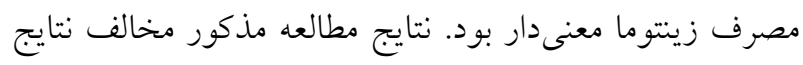

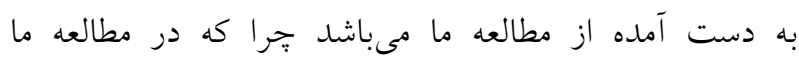

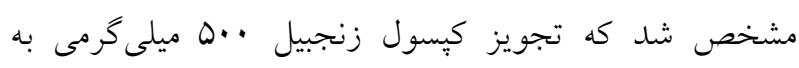

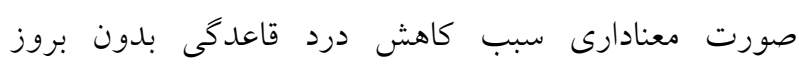

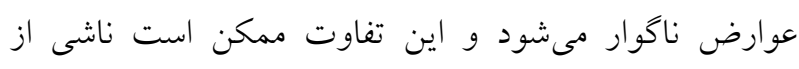

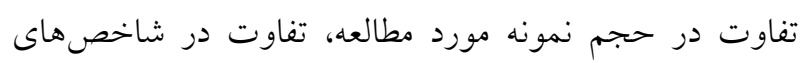

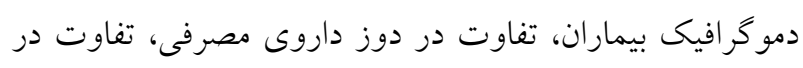

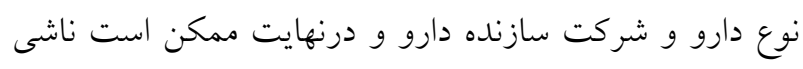

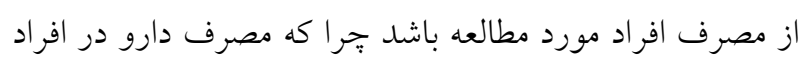

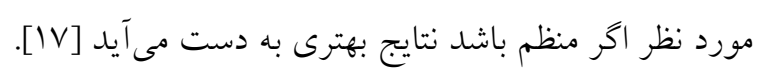

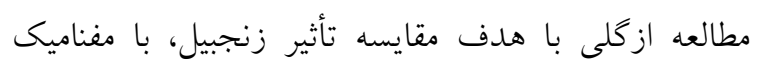

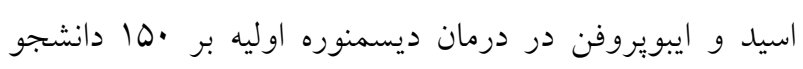




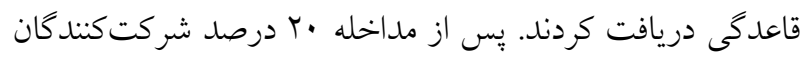

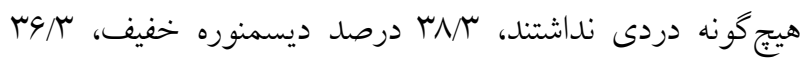
درصد ديسمنوره متوسط و ه درصد ديسمنوره شديد داشتند. اين دردين مطالعه نتيجه كيرى كرد كه زنجبيل يكى داروى مؤثر براى بهبود

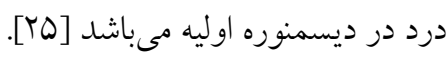

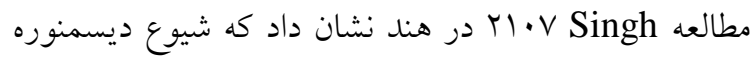

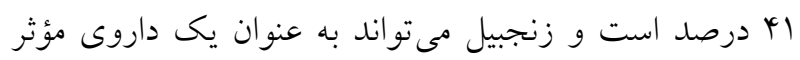

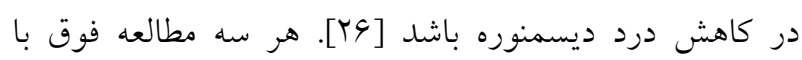
مطالعه ما همخوانى داشت.

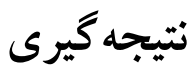

نتايج مطالعه نشان داد كه استفاده از داروى كياهى زنجبيل

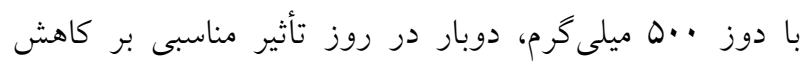

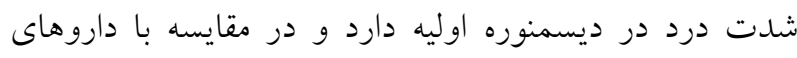

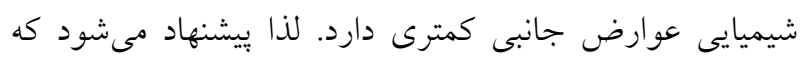

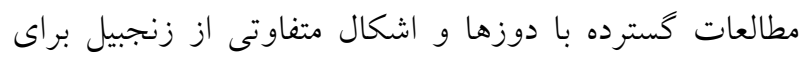

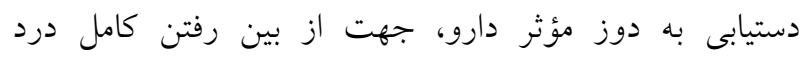

$$
\text { ديسمنوره انجام گيرد. }
$$

\section{تشكر و قدردانى}

بدينوسيله از معاونت تحقيقات و فناورى دانشخاه علوم

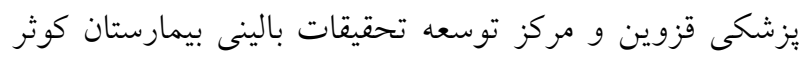
تشكر و قدردانى مى تشود.

1. Fritz MA and Speroff L. Clinical gynecologic endocrinology and infertility. 8th ed. lippincott Williams \& wilkins. Philadelphia. 2011, pp: 579.

2. Burkman RT. Berek \& Novak's gynecology. JAMA. 2012; 308 (5): 516-7.

3. Iacovides S, Avidon I and Baker FC. What we know about primary dysmenorrhea today: a critical
تسكين علائم ديسمنوره مؤثر مىباشد. بنابراين با توجه به

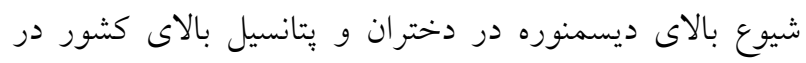

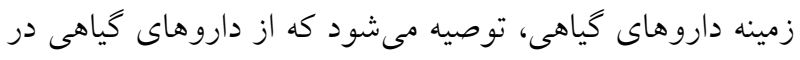

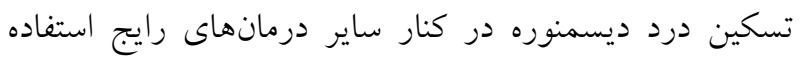

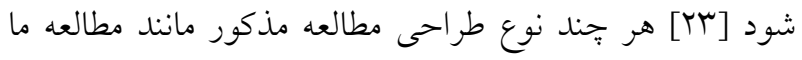

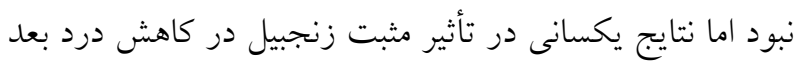
از درمان به دست آمد.

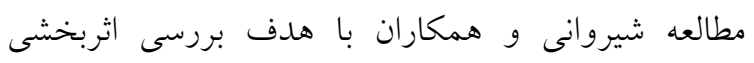

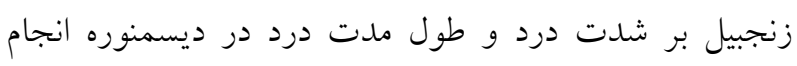

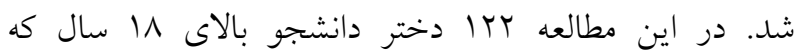

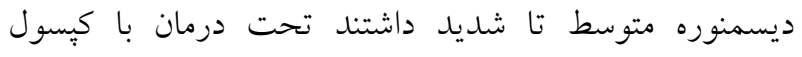

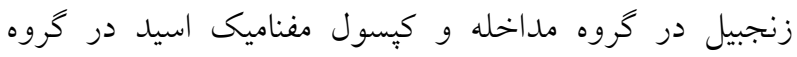

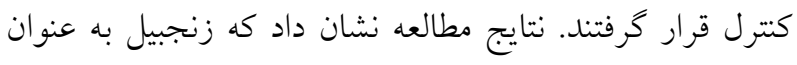

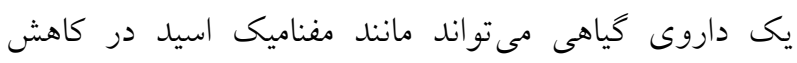

شدت درد و طول مدت درد در ديسمنوره مؤثر باشد [11)].

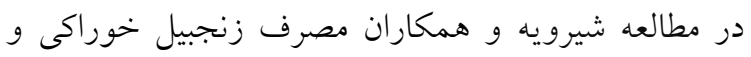

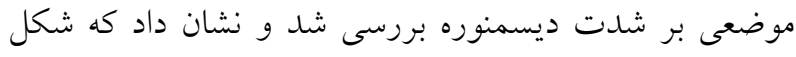

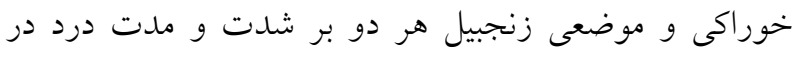

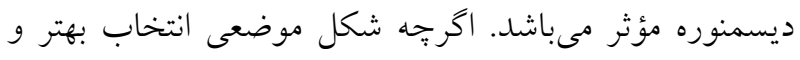

فاقد عوارض جانبى بود [YY].

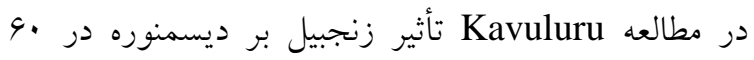
دختر نوجوان داراى ديسمنوره مورد بررسى قرار كرفت. نتايج

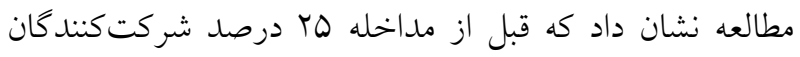

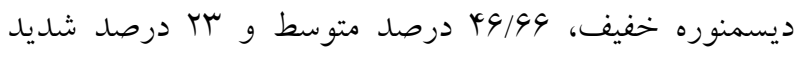

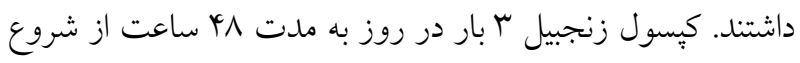

\section{منابع}

review. Human Reproduction Update 2015; 21 (6): 762-78.

4. Eden J. Dysmenorrhoea and premenstrual syndrome. Essentials of Obstetrics and Gynaecology. 1998, pp: 332-7.

5. Nayeban S, Jafarnejad F, Nayeban $S$ and Sefidgaran A. A Comparison of the Effects of Vitamin E and Vitamin B1 on the Severity and 
Duration of Pain in Primary Dysmenorrhea. $J$. Midwifery and Reproductive Health 2014; 2 (2): 143-6.

6. Akhlaghi F, Zyrak N, Nazemian S. Effect of vitamin $\mathrm{E}$ on primary dysmenorrhea. Journal of hayat. 2009; 15 (1): 13-9.

7. Davis AR and Westhoff CL. Primary dysmenorrhea in adolescent girls and treatment with oral contraceptives. J. Pediatric and Adolescent Gynecol. 2001; 14 (1): 3-8.

8. Sanfilippo $J$ and Erb T. Evaluation and management of dysmenorrhea in adolescents. Clinical Obstetrics and Gynecol. 2008; 51 (2): 257-67.

9. Eccles R, Holbrook A and Jawad M. A doubleblind, randomised, crossover study of two doses of a single-tablet combination of ibuprofen/paracetamol and placebo for primary dysmenorrhoea. Current Medical Research and Opinion 2010; 26 (11): 2689-99.

10. Kalish GM, Patel MD, Gunn ML and Dubinsky TJ. Computed tomographic and magnetic resonance features of gynecologic abnormalities in women presenting with acute or chronic abdominal pain. Ultrasound Q. 2007; 23 (3): 167-75.

11. Kamaya A, Shin L, Chen B and Desser TS. Emergency gynecologic imaging. Semin Ultrasound CT MRI. 2008; 29 (5): 353-68.

12. Fritz MA and Speroff L. Female infertility. Clinical gynecologic endocrinology and infertility. 2011, pp: 1162-3.

13. Ryan JL, Heckler CE, Roscoe JA, Dakhil SR, Kirshner J, Flynn PJ and et al. Ginger (Zingiber officinale) reduces acute chemotherapy-induced nausea: a URCC CCOP study of 576 patients. Supportive Care in Cancer 2012; 20 (7): 1479-89.

14. Kiuchi F, Iwakami S, Shibuya M, Hanaoka F and Sankawa U. Inhibition of prostaglandin and leukotriene biosynthesis by gingerols and diarylheptanoids. Chemical and Pharmaceutical Bulletin 1992; 40 (2): 387-91.
15. Ozgoli G, Goli M and Moattar F. Comparison of effects of ginger, mefenamic acid, and ibuprofen on pain in women with primary dysmenorrhea. The Journal of Alternative and Complementary Medicine 2009; 15 (2): 129-32.

16. Rahnama P, Fallah Huseini H, Mohammadi H, Modares M, Khajavi Shojaee K, Askari M and et al. The effects of Zingiber officinal R. On primary dysmenorrhea. Journal of Medicinal Plants 2010; 4 (36): 81-6.

17. Torshiz M, Golmakani N, Saadatjoo $S$ and Rakhshandeh H. Zintoma (Ginger)in the treatment of pri may dysmenorrheal. Modern Care Journal (Scientific Quarterly of Birjand Nursing \& Midwifery Faculty). 2005; 2 (3): 5-8.

18. Shirvani MA, Motahari-Tabari $\mathrm{N}$ and Alipour A. The effect of mefenamic acid and ginger on pain relief in primary dysmenorrhea: a randomized clinical trial. Archives of Gynecology and Obstetrics 2015; 291 (6): 1277-81.

19. Rahnama P, Montazeri A, Huseini HF, Kianbakht $S$ and Naseri M. Effect of Zingiber officinale R. rhizomes (ginger) on pain relief in primary dysmenorrhea: a placebo randomized trial. BMC Complementary and Alternative Medicine 2012; 12 (1): 92.

20. Kashefi F, Khajehei M, Tabatabaeichehr M, Alavinia $\mathrm{M}$ and Asili J. Comparison of the effect of ginger and zinc sulfate on primary dysmenorrhea: a placebo-controlled randomized trial. Pain Management Nursing 2014; 15 (4): 826-33.

21. Grzanna R, Lindmark L and Frondoza CG. Ginger-an herbal medicinal product with broad anti-inflammatory actions. J. Medicinal Food 2005; 8 (2): 125-32.

22. Davdabady Farahani $M$ and Seyyedzadeh Aghdam N. Comparison of ginger and valerian on the severity of primary dysmenorrhea: a randomized triple blind clinical trial. Complementary Medicine Journal of faculty of Nursing \& Midwifery 2013; 3 (2): 494-503.

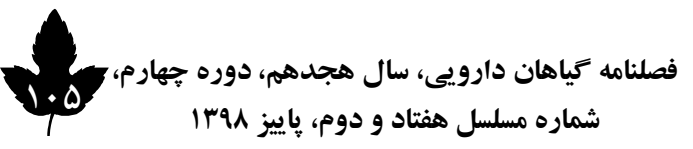


23. Samadi N, Amani F, Naghizadeh M, Alahiari I, GHezelbash S and Kazemzadeh R. Effect of Using Combination of Fennel, Chamomile and Ginger on Relieving Symptoms of Primary Dysmenorrheal among Students in Ardabil University of Medical Sciences in 2012. SJIMU. 2015; 22 (6): 159-64.

24. Shirooye P, Hashem-Dabaghian F, HamzelooMoghadam M, Afrakhteh $M$, Bioos $S$ and Mokaberinejad R. A clinical comparative study of oral and topical ginger on severity and duration of primary dysmenorrhea. Research J.
Pharmacognosy 2017; 4 (1): 23-32.

25. Kavuluru VP. A study to assess the effectiveness of ginger preparation on dysmenorrhea among adolescent girls. IJAR. 2017; 3 (3): 22-5.

26. Singh $P$, Nageshwar V and Krishnaveni R. A Study to Assess the Effectiveness of Ginger Remedy in Reduction of Dysmenorrhea among Adolescent Girls. Indian Journal of Public Health Research \& Development 2017; 8 (2): 197-200. 
بررسى تأثير زنجبيل ...

\title{
The Effect of Ginger on Primary Dysmenorrhea in Students of Qazvin University of Medical Sciences
}

\author{
Pakniat H (Ph.D.) ${ }^{1}$, Hajiseyed Javadi ES (Ph.D.) ${ }^{1^{*}}$, Golmohammadi Z (Ph.D.) ${ }^{2}$, Ashrafi M \\ (Ph.D.) $)^{2}$ \\ 1- Department of Obstetrics and Gynecology, Faculty of Faculty of Medicine, Qazvin \\ University of Medical Sciences, Qazvin, Iran \\ 2- Faculty of Medicine, Qazvin University of Medical Sciences, Qazvin, Iran \\ *Corresponding author: Kosar hospital, Taleghani Street, Qazvin, P.O.Box: 34156-13176 \\ Tel: +98-912-1822806 \\ Email: dr_seidjavadi@yahoo.com
}

\begin{abstract}
Background: Dysmenorrhea is a major individual and public health problem in women. Recently, according to the high prevalence of dysmenorrhea, different medical treatments including herbal medicines have been used.

Objective: The aim of this study is to evaluate the effect of ginger on dysmenorrhea severity in students in Qazvin University of Medical Science, Iran.

Methods: In this randomized clinical trial, 100 female students aged 18 to 25 years with moderate to severe dysmenorrhea were included. The participants were allocated to two groups of control and intervention by randomization. The intervention group were received $500 \mathrm{mg}$ of ginger tablet and $250 \mathrm{mg}$ of Mefenamic Acid capsule twice a day and the control group took placebo and Mefenamic Acid $250 \mathrm{mg}$ capsule two times a day from two days before and within the first three days of the onset of the Menstrual period. Pain intensity were measured with VAS tool before and two months after the prescription of drugs.

Results: The results of our study showed the pain intensity after the intervention in the first month, was significantly less than the control group $(3.72$ vs 5.44$)(P<0.001)$ and in the second month, the pain in intervention group was significantly less than the control group (3.2 vs5.2) $(P<0.001)$, and no complications were observed in the intervention group.

Conclusion: The result of this study indicated that herbal medicine like ginger has a great effect on reduction of dysmenorrhea severity and furthermore studies are suggested to perform in order to find a suitable dose of ginger for complete treatment of dysmenorrhea and other menstrual symptoms.
\end{abstract}

Keywords: Dysmenorrhea, Ginger, Pain

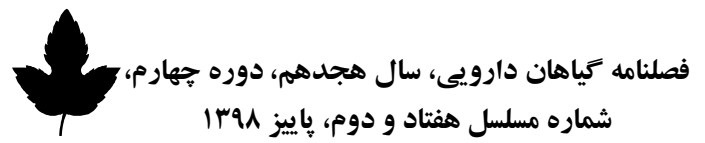

